http://dx.doi.org/10.35381/r.k.v6i11.1219

\title{
Revolución industrial 4.0: La brecha digital en Latinoamérica
}

\section{Industrial revolution 4.0: The digital divide in Latin America}

\author{
Lino Rolando Rodríguez-Alegre \\ Irrodrigueza@ucvvirtual.edu.pe$$
\text { Universidad Cesar Vallejo, Trujillo }
$$$$
\text { Perú }
$$$$
\text { https://orcid.org/0000-0002-9993-8087 }
$$$$
\text { Guido Trujillo-Valdiviezo }
$$$$
\text { gtrujillo@ucv.edu.pe }
$$$$
\text { Universidad Cesar Vallejo, Trujillo }
$$$$
\text { Perú }
$$$$
\text { https://orcid.org/0000-0002-3019-6599 }
$$ \\ Margarita Jesús Egusquiza-Rodríguez \\ megusquiza@ucv.edu.pe \\ Universidad Cesar Vallejo, Trujillo \\ Perú \\ https://orcid.org/0000-0001-9734-0244 \\ Rosario-del-Pilar López-Padilla \\ rlopezp@ucv.edu.pe \\ Universidad Cesar Vallejo, Trujillo \\ Perú \\ https://orcid.org/0000-0003-2651-7190
}

Recepción: 20 de enero 2021 Revisado: 15 de febrero 2021

Aprobación: 20 de abril 2021

Publicación: 01 de mayo 2021 


\title{
RESUMEN
}

El actual artículo de revisión tiene como objetivo analizar la revolución industrial 4.0 como brecha digital en Latinoamérica; esto con la finalidad de conocer las fortalezasdebilidades existentes desde una connotación tecnológica y social. La revolución industrial 4.0 al estar centrada desde el internet de las cosas, requiere un extremo desafío para la administración pública, en cuanto se encamina a un progresivo uso de monedas digitales basadas en blockchain. Es necesario adecuar la educación al fortalecimiento de la inteligencia artificial como factor esencial de la revolución 4.0, hasta ahora se evidencia una tímida inserción de recursos robóticos como factor educativo, siendo aun, el uso de las TIC un reto aun por consolidar.

Descriptores: Brecha digital; disparidad tecnológica; potencial científico. (Palabras tomadas del Tesauro UNESCO).

\begin{abstract}
The current review article aims to analyze the industrial revolution 4.0 as a digital divide in Latin America; this in order to know the existing strengths-weaknesses from a technological and social connotation. The industrial revolution 4.0, being centered from the internet of things, requires an extreme challenge for the public administration, insofar as it is heading towards a progressive use of digital currencies based on blockchain. It is necessary to adapt education to the strengthening of artificial intelligence as an essential factor of the 4.0 revolution, until now there is a timid insertion of robotic resources as an educational factor, the use of ICT still being a challenge yet to be consolidated.
\end{abstract}

Descriptors: Digital divide; technological gap; scientific potential. (Words taken from the UNESCO Thesaurus). 


\section{INTRODUCCIÓN}

Las últimas décadas del siglo $\mathrm{XX}$ y primeras del $\mathrm{XXI}$, han desencadenado el uso del internet como factor esencial para la comunicación, desarrollándose un progresivo proceso de adaptación al uso de la tecnología conectada a la red ciberespacial. A juicio de (Papí-Gálvez \& Escandell-Poveda, 2019), el principal reto de la revolución del internet, radica en que precisamente debe ser trabajado en concordancia con los entornos digitales, los cuales deben ser operados por personas con poca y máxima pericia, en combinación de lograr la efectividad planteada en la estrategia comunicacional.

En este sentido, el internet se constituye en la base para la transferencia de conocimientos (Andrade-Mendoza, 2019), por medio de un proceso comunicacional global donde se articula la esencia para confrontar la era industrial 4.0, proyectándose esta como el presente educativo, comercial, económico, comunicativo; es decir, se asientan las bases para una sociedad digital enfocada desde la inteligencia artificial como eje fundamental de la convivencia humana con la robótica, sin embargo, se vierte una seria amenaza de sustitución de la humanidad por los robots como operadores para desarrollar diversos trabajos, focalizándose así, mayor desempleo y pérdida de calidad de vida de los diversos grupos sociales (Vila-De-Prado, 2019).

Esta advertencia debe ser tenida en cuenta, por cuanto se juega la estabilidad de la familia como núcleo central de la sociedad, lo cual es inevitable por el avance disruptivo de la industria 4.0, significando la total automatización de los procesos industriales con la excusa de mayor producción a bajo costo, sin embargo, de llegar a existir una masificación de este modelo productivo, es correlativo a mayor desempleo-pobreza; por tal motivo, el actual papel de trabajo desde una vertiente analítica, tiene como objetivo analizar la revolución industrial 4.0 como brecha digital en Latinoamérica; esto con la finalidad de conocer las fortalezas-debilidades existentes desde una connotación tecnológica y social. 


\section{Industria 4.0 su génesis global}

Este modelo industrial tiene íntima relación con el paradigma digital al estar basada primordialmente relacionado con la aplicación de la tecnología e inteligencia artificial como fundamento epistémico, siendo operacionalizada aun, por una combinación de humano y máquina, siendo Alemania uno de los primeros países en implementar tal avance, especialmente en perfeccionar la industria manufacturera, acotándose que tal avance no ha sido al azar, sino, derivada de políticas públicas bien organizadas y aplicadas en combinación de acción de los ministerios de educación e industria (Casalet, 2018).

A juicio de la precitada autora, China es el otro país que ha tenido mayores avances, por el desarrollo tecnológico y comercio digital que adelanta como proceso de globalización del mercado; sumándose Estados Unidos con el sector de manufactura, impulsada por medio de la nanotecnología, otros países que han cooperado en la generación industrial 4.0, se encuentra Japón, Italia, por lo que la cooperación internacional juega un papel importante en la consolidación de este enfoque digital.

En este primer segmento de nacimiento de la 4.0, se evidencia la necesidad de contar con políticas publicas claras y encaminadas a fortalecer un segmento industrial que aún puede percibirse lejano en Latinoamérica como si fuese de una película futurista, pero es el presente en algunas naciones donde se viene trabajando en cooperación para fortalecer un nuevo modelo. En este sentido, la educación con la vinculación industrial en conjugación de la investigación, ha sido el factor esencial que ha trabajado Alemania para favorecer una mano de obra calificada, la cuales hasta ahora no se ha visto amenazada, sino, fortalecida en los beneficios económicos y sociales adquiridos, en este sentido, es necesario revisar el enfoque de educación dual desarrollado por este país para comprender la base del éxito que hasta ahora promulgan (Flores-Sánchez \& Vigier, 2020). 


\section{Revolución 4.0 y educación dual}

El desarrollo de estas variables tiene su funcionabilidad en gestionar ciudades basadas en el internet, destacándose que esta iniciativa ha figurado en los países que tradicionalmente han estado en la vanguardia tecnológica, investigación e innovación (Do-Nascimento-João, et al. 2019), los autores brindan luces sobre la necesidad que se tiene en Latinoamérica de gestionar un acceso equitativo, de calidad, al internet, así como profundizar la investigación e innovación dentro del currículo educativo en todos sus niveles, especialmente en el universitario donde se hace pertinente diseñar un modelo integrador entre academia-industria con base a lo público-privado.

Alemania por ser el país patrocinante de la industria 4.0, por así decirlo, ha sabido configurar lo planteado anteriormente, logrando a través de su educación dual, la formación académica y laboral de la mano, siendo la empresa un campo de entrenamiento, mientras que la universidad se encarga de promover la formación cognitiva, así como competencias ciudadanas, así ambas instituciones se complementan a la luz de políticas de gobierno estructuradas para ser desarrolladas eficazmente en beneficio de la población (Alemán-Falcón, 2015).

Este modelo educativo no ha sido improvisado, es producto de la planificación y trabajo de varias décadas, quizás se encuentre hoy brindando frutos con efectividad, siendo este un rasgo a estudiar para que pueda ser aplicado en Latinoamérica con mayor eficiencia. En Ecuador se viene manejando esta experiencia en los últimos años, lo que se puede señalar como los primeros pasos, siendo indispensable contar con un aprendizaje experiencial en el "saber hacer" (Juanes-Giraud \& Rodríguez-Hernández, 2020), lo cual confirma la necesidad de transcender hacia un modelo educativo holístico con visión global de la productividad del aprendizaje en concordancia con lo económico, implicando trabajar desde el abordaje político - educativo, encontrándose en esta área, una amenaza para establecer la industria 4.0 en la región con efectividad, dado a la polarización ideológica reinante en los últimos años en la región, lo cual, no debería retrasar que las universidades adopten un modelo flexible de formación en competencias 
no solo para el saber, sino, para el hacer (Echeverría-Samanes \& Martínez-Clares, 2018). Prosiguiendo con la experiencia ecuatoriana, (Espinoza-Freire, 2020), advierte que este enfoque educativo no debe percibirse como una mera práctica pre profesional, sino, como un estilo educativo que fusiona lo teórico con lo práctico en donde dos instituciones se encargan de la formación integral del aprendiz, siendo necesario fortalecer su operatividad en concordancia con lo planteado por (Echeverría-Samanes \& MartínezClares, 2018), confirmándose la existencia de vacíos procedimentales que deben ser mejorados con la finalidad de lograr la efectividad deseada para el beneficio de la sociedad, siendo indispensable repensar el currículo educativo no solo en el papel, sino, en el hacer, siendo necesaria un cambio de mentalidad para tal fin En México se viene aplicando la educación dual como política de Estado desde el 2013, a juicio de (Zamora-Torres \& Thalheim, 2020), esta tiene su esencia en procura de disminuir la brecha de desempleo, la cual principalmente existe en la población joven recién graduada de la escuela o universidad, en la investigación de los investigadores precitados se destaca una correlación positiva en consideración que este enfoque educativo ha contribuido en disminuir la brecha del desempleo. 


\section{Touste Mexicano de Formación Dual}

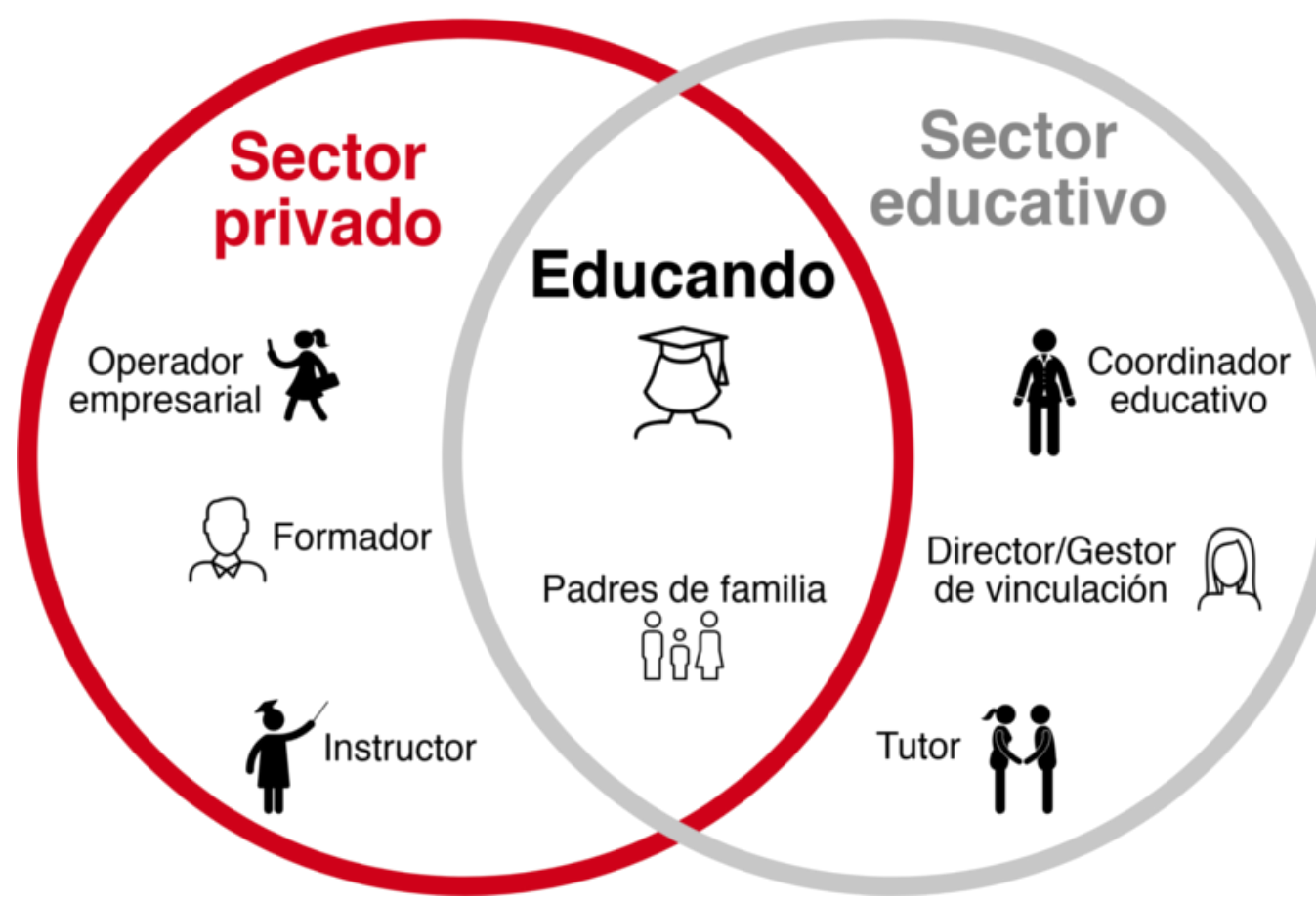

Figura 1. Modelo mexicano de formación dual.

Fuente: Cámara Suizo-Mexicana (2020).

Desde 2016 existe la alianza entre Suiza y México para aplicar el modelo suizo de educación dual en el bachillerato mexicano, así los estudiantes optan por un modelo tecnológico que permite combinar la educación formal con la preparación al trabajo directamente en las empresas, siendo que la familia interviene directamente en el acompañamiento del aprendiz en el proceso educativo - formativo, siendo aun, temprano para dilucidar la efectividad de esta apuesta por transcender a un modelo dual educativo. Por otro lado, la investigación de (Flores-Sánchez \& Vigier, 2020), indica que la educación dual proporciona un salario promedio de $275 \$$ durante la estadía en el programa, mientras que los egresados de este modelo logran una plaza de empleo más rápido que quienes 
provienen del modelo tradicional.

En Colombia a juicio de (Rojas, 2015), el modelo ha sido positivo, siendo necesario intensificar su aplicación a una proporción mayor poblacional con la intención de proyectar mayor posibilidad de estar debidamente entrenados para sumarse al campo laboral de modo efectivo, sobre todo cuando el empleado percibe al estudiante como un catalizador importante entre la teoría y práctica (Gutiérrez-Salazar \& Darío-Barrero, 2015).

La investigación de (Porta-Castillo, 2020), indica que en el Perú, esta experiencia ha sido satisfactoria en el reducido segmento poblacional donde se ha implementado, siendo necesario profundizar no solo en masificar a otras instituciones, sino, diseñar una política educativa factible y viable que contribuya al desarrollo efectivo en los próximos años de esta modalidad, para lo cual se sugiere estudiar la propuesta alemana y suiza por cuanto ambas se desarrollan con fortaleza en el sector universitario y bachillerato, para lo cual se requiere de mayor participación ciudadana para conciliar tal proyecto en beneficio de reducir las brechas sociales.

Siendo necesario tener en cuenta la propuesta de (Sadie-Smeck \& Fiszbein, 2020), sobre los siete retos que tiene Latinoamérica para lograr una educación dual efectiva, entre las cuales se hace mención:

Desafío \#1: Regulaciones débiles relativas a la relación empleador-aprendiz (por ejemplo, contrato, compensación)

Desafío \#2. Falta de normas y bajo reconocimiento del valor de la formación

Desafío \#3: Normas y mecanismos débiles para hacer cumplir las regulaciones y normas Desafío \#4: Falta de coordinación entre los actores

Desafío \#5: Pocos incentivos para la participación en las empresas y la cofinanciación

Desafío \#6: Baja integración de personas con mayor necesidad

Desafío \#7: Escasa evidencia para la toma de decisiones

Cada uno de los desafíos mencionados, requieren de una voluntad política en articular las leyes en concordancia con propiciar la integración de la educación y desarrollo 
empresarial como un ente en donde se trabaja coordinadamente, garantizando el derecho a la educación y al trabajo, por lo que los empleadores, así como aprendices, deben tener garantía contractual, lo cual es fundamental desde una reinserción de los impuestos a la generación de un modelo dual favorable para el crecimiento integral, sin embargo, el tema de aranceles suele ser una de las mayores que posee la región (BritoGaona \& Iglesias, 2017).

\section{Revolución 4.0 y brecha digital en Latinoamérica}

Una de las principales brechas a nivel 4.0 se encuentra en la administración pública (Llanes-Font, et al. 2020), por cuanto la figura del Estado es el mayor ente o empleador de la región, así como el proveedor de los servicios públicos, lo cual requiere de una política de formación del empleado publico en relación de aprender no en este caso a operar un dispositivo tecnológico-informático, sino, lo relacionado a la inteligencia artificial y robótica, generándose una brecha que califica el orden diferencial al cual se encuentra la región.

En este sentido, (Montecinos, 2021), comenta que el sector de la administración pública es el que posiblemente se vea en mayor afección a causa de depender de lineamientos gubernamentales basados en políticas que aun no se reflejan en contundencia, lo cual podría conducir a la improvisación de aplicar la $4.0 \mathrm{sin}$ un debido respaldo socio económico en protección del trabajador, causando mayor brecha en la pobreza existente. 
Indudablemente que la debilidad del sector público, debe ser superada por el privado, sin embargo, la plataforma digital suele ser débil en la región, generándose brechas entre quienes tienen acceso al internet y quienes no (ver figura 2).

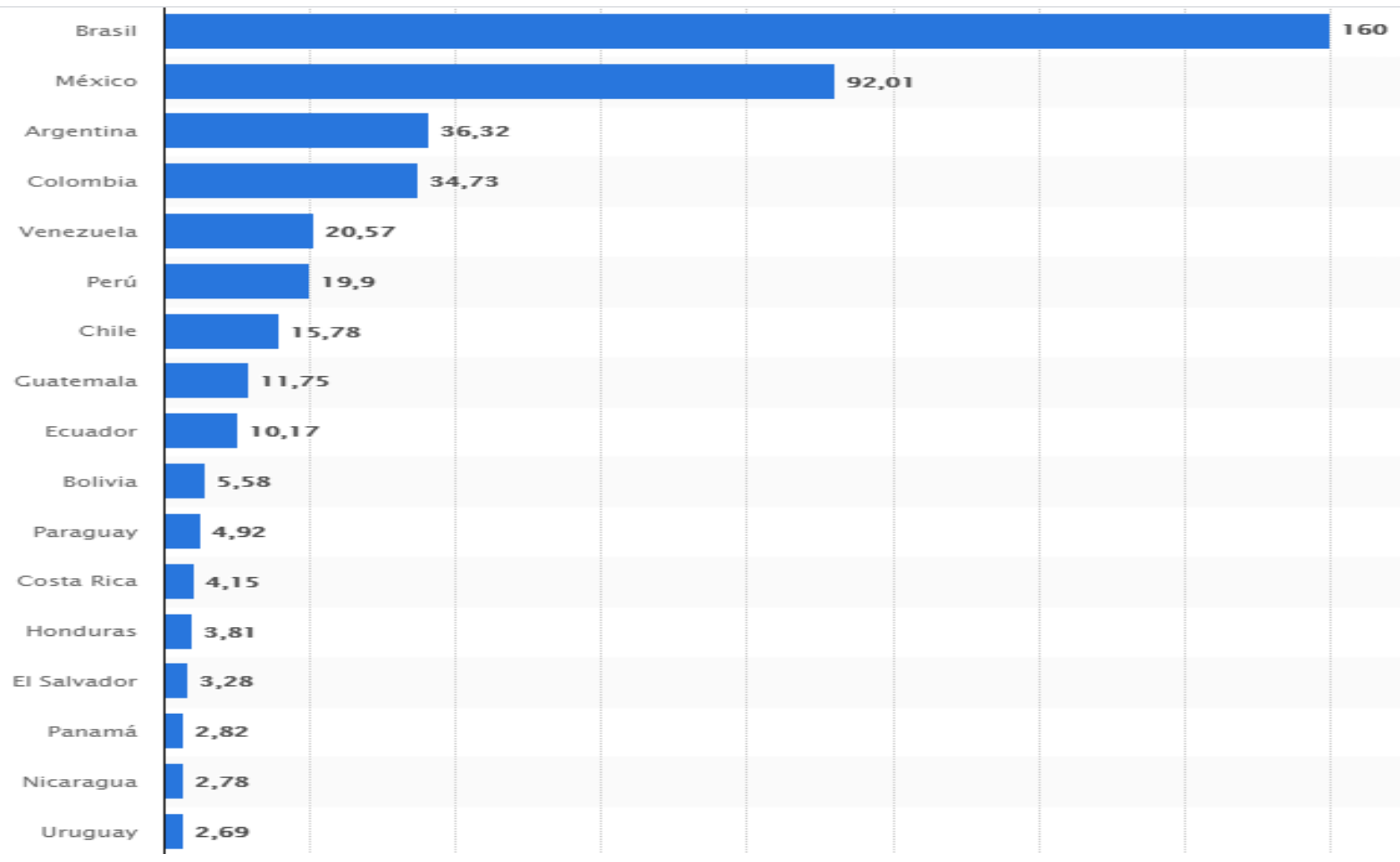

Figura 2. Número de usuarios de internet por país en América Latina en enero de 2021. Fuente: Statista (2021).

La figura evidencia una desproporción estadística entre las personas que tienen acceso a internet y lo usan, lo cual evidencia una brecha entre los países de la región, esto sin estudiar los factores internos de cada país que originan tal desigualdad, este desequilibrio debe ser minimizado en requerimiento de contar con una industria 4.0 favorable, por cuanto se corre el riesgo de incrementar la pobreza y desigualdades si esta se aplica por iniciativa de algunos países y no como un bloque uniforme a la luz de políticas viables para tal fin. 
Por otro lado, (Llanes-Font, et al. 2020) indican que uno de los principales retos, es la aplicación transversal en la administración pública de las herramientas digitales con la finalidad de permear las políticas a aplicar en beneficio de instaurar efectivamente la era 4.0 en el escenario latinoamericano y caribeño, lo cual no debe confundirse con la revolución 3.0 basada en la informática, para lo cual, se brinda la siguiente infografía (ver figura 3):

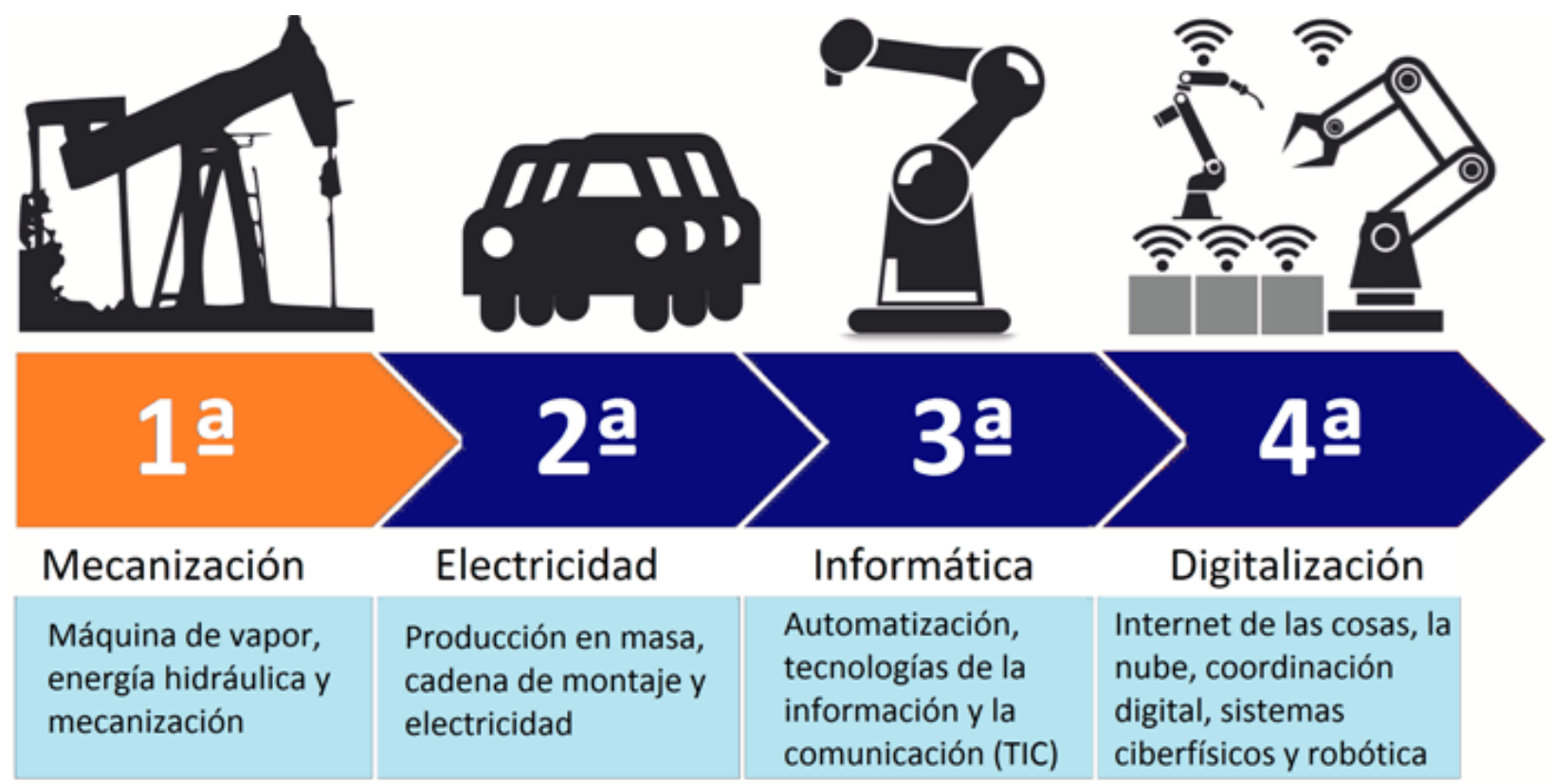

Figura 3. Revolución industrial.

Fuente: Vicent-Selva-Belén (2016).

Por lo tanto, la revolución industrial 4.0 al estar centrada desde el internet de las cosas, requiere un extremo desafío para la administración pública, en cuanto se encamina a un progresivo uso de monedas digitales basadas en blockchain, lo cual, evidentemente modificará la legislación, por cuanto se deberá penalizar delitos relacionados al ciberespacio, como hackeo monetario, de identidad, entre otros que puedan surgir en la naciente era digital (Márquez-Díaz, 2019), lo cual indudablemente involucra el factor 
bioético como factor de análisis para el derecho, siendo un llamado a los legisladores a no ignorar el creciente estilo de vida basado en lo tecnológico-digital, por cuanto deberán legislar en función de una sociedad basada en la era de la internet (García-Segura \& Cayón-Peña, 2019), siendo requerido para tal fin, la participación de todos los factores que conforman la sociedad (Aldana-Zavala, et al. 2021).

\section{CONCLUSIÓN}

En la Latinoamérica existen brechas para la consolidación de la industria 4.0 en el corto y mediano plazo, por cuanto se requiere de la participación activa de todos los actores que conforman la sociedad, involucrando lo público-privado, en razón de diseñar políticas públicas que contribuyan al establecimiento de la era digital como eje transversal de convivencia social.

Es necesario adecuar la educación al fortalecimiento de la inteligencia artificial como factor esencial de la revolución 4.0, hasta ahora se evidencia una tímida inserción de recursos robóticos como factor educativo, siendo aun, el uso de las TIC un reto aun por consolidar, más aún, cuando la investigación e innovación debe adaptarse a escenarios de preparación de los estudiantes para asumir los retos digitales; aunque en este campo se ha iniciado el desarrollo de la educación dual, sin embargo, no se percibe como suficiente en su accionar, por cuanto se requiere de mayor actualización de las empresas al ambiente 4.0 para que pueda generarse un complemento formativo en las empresas. Se ha evidenciado en las experiencias documentadas, que la educación dual se ha generado en razón de mejorar la brecha de desempleo, siendo necesario producirla en razón desde aprender a manejar efectivamente el internet de las cosas, por cuanto básicamente el empleo del futuro inmediato es basado lo digital.

Es necesario fortalecer el sistema educativo en base a lo dual-digital, como premisa de minimizar brechas de cara al futuro donde se proyecta mayor desempleo, si no se está preparado para laborar en una sociedad basada en un comercio digital, teniéndose en cuenta que Alemania ha implementado un proceso sistemático y progresivo de educación 
dual, a la par de la revolución 4.0, lo cual, en ambos escenarios los coloca en el top mundial, siendo necesario estudiar su crecimiento, sobre todo en compaginar las políticas publicas con lo privado, siendo este tema aun, lejos de concretar en Latinoamérica. Latinoamérica debe inminente superar las brechas de pobreza y de acceso al internet en lo inmediato, a la par de consolidar la entrada de la era 4.0, sino se corre el riesgo de estar relegados y en producción de mayor pobreza a la luz del desempleo, siendo necesario un cambio paradigmático para el abordaje educativo en razón de aprender a hacer en apoyo de estrategias sostenibles que transciendan el modelo de consumo digital al de producción que tanta falta le está haciendo a la región.

\section{FINANCIAMIENTO}

No monetario.

\section{AGRADECIMIENTO}

A la Universidad Cesar Vallejo, Trujillo; por motivar el desarrollo de la investigación.

\section{REFERENCIAS CONSULTADAS}

Aldana-Zavala, J, Vallejo-Valdivieso, P, \& Isea-Argüelles, J. (2021). Investigación y aprendizaje: Retos en Latinoamérica hacia el 2030 [Research and learning: Challenges in Latin America towards 2030]. ALTERIDAD. Revista de Educación, 16(1), 78-91. https://doi.org/10.17163/alt.v16n1.2021.06

Alemán-Falcón, J. (2015). El sistema dual de formación profesional alemán: escuela y empresa [The dual system in the German vocational training: school and enterprise]. Educação e Pesquisa, 41(2), 495-511. https://doi.org/10.1590/s1517$\underline{97022015021532}$

Andrade-Mendoza, K. (2019). Comunicación digital en desarrollo turístico de la Isla Puná, Ecuador [Digital communication as a tourism development of Puná Island, Ecuador]. Universitas, Revista de Ciencias Sociales y Humanas, (30), 211227. https://doi.org/10.17163/uni.n30.2019.10 
Brito-Gaona, L, \& Iglesias, E. (2017). Inversión privada, gasto público y presión tributaria en América Latina [Private investment, government expenditure and tax burden in Latin America]. Estudios de economía, 44(2), 131156. https://dx.doi.org/10.4067/S0718-52862017000200131

Cámara Suizo-Mexicana (2020). Educación dual [Dual education]. [Internet].

Casalet, M. (2018). La digitalización industrial: un camino hacia la gobernanza colaborativa. Estudios de casos [Industrial digitization: a path towards collaborative governance. Case studies]. Documentos de Proyectos (LC/TS.2018/95), Santiago, Comisión Económica para América Latina y el Caribe (CEPAL). https://n9.cl/1eya

Do-Nascimento-João, B, Lobo-De-Souza, C, \& Serralvo, F. (2019). A systematic review of smart cities and the internet of things as a research topic. Cadernos EBAPE.BR, 17(4), 1115-1130. https://doi.org/10.1590/1679-395174442x

Echeverría-Samanes, B, \& Martínez-Clares, P. (2018). Revolución 4.0, Competencias, Educación y Orientación [Revolution 4.0, Skills, Education and Guidance]. Revista Digital de Investigación en Docencia Universitaria, 12(2), 434. https://dx.doi.org/10.19083/ridu.2018.831

Espinoza-Freire, E. (2020). La formación dual en Ecuador, retos y desafíos para la educación superior y la empresa [Dual training in Ecuador, challenges and challenges for higher education and business]. Revista Universidad $y$ Sociedad, 12(3), 304-311.

Flores-Sánchez, G, \& Vigier, H. (2020). El impacto del modelo educativo dual en la formación profesional del estudiante [The Impact of the Dual Educational Model on the Student's Professional Training]. Revista Colombiana de Educación, (78), 173206. https://doi.org/10.17227/rce.num78-9535

García-Segura, L., \& Cayón-Peña, J. (2019). Retos jurídicos de los vehículos conectados en la era del internet de las cosas [Legal challenges of connected vehicles in the era of the internet of things]. Boletín mexicano de derecho comparado, 52(154), 457-488. Epub 12 de mayo de 2020.https://doi.org/10.22201/iij.24484873e.2019.154.14150 
Gutiérrez-Salazar, A, \& Darío-Barrero, J. (2015). Análisis del impacto del modelo de educación dual en las empresas formadoras del departamento del Quindío en el año 2013 [Analysis of the impact of the dual education model in companies trainers from the department of Quindío in 2013]. Titulación MBA. Recuperado de https://n9.cl/bd7d3

Juanes-Giraud, B, \& Rodríguez-Hernández, C. (2020). La formación dual. Elementos de análisis para implementación en una universidad ecuatoriana [Dual training. Analysis elements for implementation in an Ecuadorian university]. Conrado, 16(74), 354-363.

Llanes-Font, M, Díaz-de-Ceballos, M, Salvador-Hernández, Y. (2020). Administración pública y cuarta revolución industrial. ¿Qué nos lleva hasta allí? [Public administration and the fourth industrial revolution. What gets us there?]. XXXIII Concurso del CLAD sobre Reforma del Estado y Modernización de la Administración Pública. Recuperado de https://n9.cl/copv3

Llanes-Font, M, Salvador-Hernández, Y, Suárez-Benítez, M, \& Solórzano-Benítez, R. (2020). Cuarta revolución industrial y administración pública de América Latina y el Caribe [Fourth industrial revolution and public administration in Latin America and the Caribbean]. Ciencias Holguín, 26(3),78-92.

Márquez-Díaz, J. (2019). Riesgos y vulnerabilidades de la denegación de servicio distribuidos en internet de las cosas [Risks and vulnerabilities of the denial of service distributed on the internet of things]. Revista de Bioética y Derecho, (46), 85-100.

Montecinos, E. (2021). Cuarta revolución industrial y la administración pública en América Latina [Fourth industrial revolution and public administration in Latin America]. Revista Venezolana De Gerencia, 26(93), 10-32. https://doi.org/10.52080/rvgluz93.02

Papí-Gálvez, N, \& Escandell-Poveda, R. (2019). Retos de internet del futuro y generación española no digital: datos nacionales 2017 [Challenges for the internet of future and non-digital Spanish generation: national data 2017]. Cuadernos.info, (45), 173-190. https://dx.doi.org/10.7764/cdi.45.1524

Porta-Castillo, C. (2020). Formación profesional dual como factor importante para la productividad laboral [Dual vocational training as an important factor for labor productivity]. Trabajo de titulación. [Internet]. Recuperado de https://n9.cl/fflki 
Rojas, L. (2015). La formación dual en Colombia. El caso de la Fundación Universitaria de la Cámara de Comercio de Bogotá Uniempresarial: los desafíos actuales en la percepción de egresados y empresarios [The Colombian Dual Apprenticeship System: the Case of the Business University Foundation of the Chamber of Commerce of Bogotá Uniempresarial: The Present Challenges in the Perception of Graduat es and Businessmen]. Rev. Cient. Gen. José María Córdova 13(15), 145-181.

Sadie-Smeck, M, \& Fiszbein, A. (2020). Educación Dual en América Latina Desafíos y oportunidades [Dual Education in Latin America Challenges and opportunities]. Washington, DC, Diálogo Interamericano. https://n9.cl/7rs7a

Statista (2021). Número de usuarios de internet por país en América Latina en enero de 2021 [Number of internet users by country in Latin America in January 2021]. https://n9.cl/8762j

Vicent-Selva-Belén, J. (2016). Revolución industrial [Industrial Revolution]. Recuperado de https://n9.cl/ci9bo

Vila-De-Prado, R. (2019). Consecuencias económicas y sociales de la cuarta revolución industrial y estrategias pensadas para la adaptación de la actividad económica [ Economic and social consequences of the fourth industrial revolution and strategies intended for the adaptation of economic activity]. Revista Aportes de la Comunicación y la Cultura, (26), 89-108.

Zamora-Torres, A, \& Thalheim, L. (2020). El Modelo Mexicano de Formación Dual como modelo educativo en pro de la inserción laboral de los jóvenes en México [The Mexican Dual Training Model as an educational model for the insertion of young people into the labor market in Mexico]. Revista iberoamericana de educación superior, 11(31), 48-67. https://doi.org/10.22201/iisue.20072872e.2020.31.705

C2021 por los autores. Este artículo es de acceso abierto y distribuido según los términos y condiciones de la licencia Creative Commons Atribución-NoComercial-Compartirlgual 4.0 Internacional (CC BY-NC-SA 4.0)

(https://creativecommons.org/licenses/by-nc-sa/4.0/). 